\title{
Circular Dichroism in Biological Photonic Crystals and Cubic Chiral Nets
}

\author{
M. Saba, ${ }^{1}$ M. Thiel, ${ }^{2}$ M. D. Turner ${ }^{3}$ S. T. Hyde,${ }^{4}$ M. Gu, ${ }^{3}$ K. Grosse-Brauckmann, ${ }^{5}$ D. N. Neshev, ${ }^{6}$ \\ K. Mecke, ${ }^{1}$ and G. E. Schröder-Turk ${ }^{1, *}$ \\ ${ }^{1}$ Theoretische Physik, Friedrich-Alexander Universität Erlangen-Nürnberg, 91058 Erlangen, Germany \\ ${ }^{2}$ Angewandte Physik \& Center for Functional Nanostructures, Karlsruhe Institute of Technology, 76128 Karlsruhe, Germany \\ ${ }^{3}$ Centre for Micro-Photonics \& CUDOS, Swinburne University of Technology, VIC 3122, Australia \\ ${ }^{4}$ Applied Mathematics, Research School of Physics and Engineering, Australian National University, Canberra, Australia \\ ${ }^{5}$ Fachbereich Mathematik, Technische Universität Darmstadt, 64289 Darmstadt, Germany \\ ${ }^{6}$ Nonlinear Physics Centre, Research School of Physics and Engineering, Australian National University, Canberra, Australia
}

(Received 5 November 2010; revised manuscript received 27 January 2011; published 11 March 2011)

\begin{abstract}
Nature provides impressive examples of chiral photonic crystals, with the notable example of the cubic so-called srs network (the label for the chiral degree-three network modeled on $\mathrm{SrSi}_{2}$ ) or gyroid structure realized in wing scales of several butterfly species. By a circular polarization analysis of the band structure of such networks, we demonstrate strong circular dichroism effects: The butterfly srs microstructure, of cubic $I 4_{1} 32$ symmetry, shows significant circular dichroism for blue to ultraviolet light, that warrants a search for biological receptors sensitive to circular polarization. A derived synthetic structure based on four like-handed silicon srs nets exhibits a large circular polarization stop band of a width exceeding $30 \%$. These findings offer design principles for chiral photonic devices.
\end{abstract}

DOI: 10.1103/PhysRevLett.106.103902

Photonic crystals (PCs) based on the geometry of oriented triply-periodic gyroid surfaces [1] have been found in wing scales of several butterfly species [2-4], see Fig. 1. This consolidated chitin net has been linked to structural color [5]. As we show here, chirality causes circular dichroic effects, that may relate to insect communication. In contrast to human vision, many arthropods can detect linearly polarized light [6]. Sensitivity to circularly polarized light is less well understood; Gonodactylus smithii (mantis shrimps) respond to distinct states of circularly polarized light [7]; Chrysina gloriosa (jewel beetle) are distinctly more brilliant in left-handed circularly polarized (LCP) than in right-handed circularly polarized (RCP) light [8]; behavioral studies suggest sensitivity of the butterfly Heliconius cydnois to circular polarization [9]. These findings suggest that self-assembled biological structures are worth exploring for hints to the optimal design of synthetic chiral optical metamaterials and circular polarization-sensitive optical devices [10-12]. Here we reveal the origin of the impressive circular dichroism of chiral 1 srs and 4 srs geometries by a circular polarization analysis of the band structure.

The srs net [13], also known as (10,3)-a or Laves' graph $[14,15]$, is a chiral network with space group $I 4_{1} 32$ [16], named after the $\mathrm{Si}$ network in the polycationic $\mathrm{SrSi}_{2}$ crystal [18]. The srs net has a fourfold screw helix which projects to squares along [100] and a threefold screw helix, of opposite handedness, that forms triangles in projection along [111]. We call a right-handed (RH) srs net one whose threefold helices are RH. Decoration of the edges of an srs net by a body, or scaffold, with a bounding surface of constant mean curvature $H$ (corresponding to inflation
PACS numbers: 42.70.Qs, 42.79.Ci, 78.20.Bh, 81.05.Xj

with constant pressure according to the Young-Laplace equation) provides a continuous set of bodies of varying volume fraction $\Phi(H)$ [19]. The special case with $H=0$ and $\Phi=0.5$ is Schoen's gyroid minimal surface [1] that bisects space into a pair of identical but enantiomeric labyrinths, represented by a left-handed (LH) and a $\mathrm{RH}$ srs net, respectively. Realized as an achiral sheet of finite thickness, it is ubiquitous in self-assembled materials [3]. Materials structured according to a chiral single srs net are less common, but have been reported in zeolites [20], terblock-copolymers [21] and butterfly wing scales [2-4].

The most symmetric filling of the complementary volume of an inflated single srs net is its enantiomer, resulting
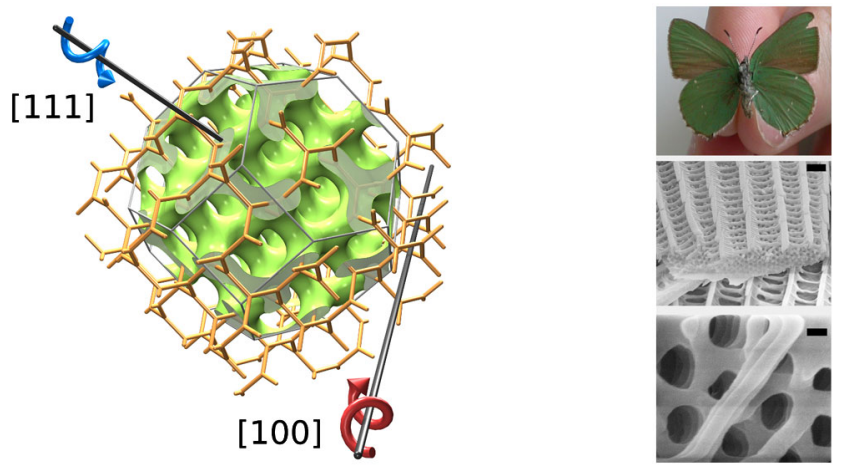

FIG. 1 (color online). Gyroid structure (see supplemental material [39] for animation). (Left) 1srs network and CMC surface with $\Phi=0.18$ clipped to a truncated octahedron. The chitin domain has a LH three-screw along [111] and a RH four-screw along [100], perpendicular to the truncated octahedron's faces. (Right) Callophrys rubi and SEM images of its wing scale. The scale bars are $1 \mu \mathrm{m}$ (middle) and $100 \mathrm{~nm}$ (bottom). 
in the achiral double gyroid ( $I a \overline{3} d$, no. 230 [17]). Curiously, space can be also symmetrically filled with multiple srs nets of the same handedness, forming polycontinuous chiral patterns. A face-centered cubic lattice can be described by the vertex positions of a quartet of intergrown like-handed srs nets [15], synthesized in metalorganic frameworks [22]. Later studies established that three, four, and eight srs nets (of equal handedness) can be intergrown [23], with chiral cubic symmetry [24-26]. The single and quadruple srs patterns are denoted 1srs and 4srs, respectively.

Circular dichroism index.-We propose an essential improvement of the method by Lee and Chan [27] to analyze circular dichroism in spatially complex PCs. Propagation of incident circularly polarized light into chiral PCs can be analyzed by scattering matrix methods [28] and by finite element and difference methods [29]; these techniques predict the absorption and reflectance rates, but the underlying structural causes are better elucidated by analysis of the band structure. However, band structure computations (by frequency domain methods as implemented in the MIT Photonic Bands package [30]) do not directly yield transmission or reflectance spectra, but this information can be extracted from these band structure data by the method detailed here.

In an infinite $\mathrm{PC}$ where the dielectric constant is a periodic function $\epsilon(\mathbf{r})$, the time-averaged magnetic field solutions are Bloch modes $\mathbf{H}_{\mathbf{k}, n}(\mathbf{r})=\sum_{\mathbf{G}} \mathbf{h}_{\mathbf{G}} e^{i(\mathbf{k}+\mathbf{G}) \cdot \mathbf{r}}$ with frequency dispersion relations $\omega_{n}(\mathbf{k})$. The degree of circular polarization is measured by the overlap integrals

$$
C_{\mathbf{k}, n}^{ \pm} \propto\left|\sum_{\mathbf{G}}\left(\mathbf{e}_{x} \pm i \mathbf{e}_{y}\right) \cdot \mathbf{h}_{\mathbf{G}} \int_{P} d x d y e^{i \mathbf{G} \cdot \mathbf{r}}\right|^{2}
$$

between the Bloch modes and an incident circularly polarized plane wave with wave vector $\mathbf{q} \| \mathbf{k}=k \mathbf{e}_{z} . P$ is the cutoff plane of the crystal, also perpendicular to $\mathbf{e}_{z}$. Equation (1) corresponds to the equation on p. 8087 of Ref. [27], in Fourier representation. Evaluation in Fourier space is substantially more efficient because of $\int d x \exp \left(\imath G_{x} x\right) \propto \delta\left(G_{x}\right)$ and $\int d y \exp \left(\iota G_{y} y\right) \propto \delta\left(G_{y}\right)$. Assuming that a plane wave with wave vector $\mathbf{q}$ does not couple to a mode $\mathbf{H}_{\mathbf{k}, n}$ with group velocity in the opposite direction $\left(\nabla_{k} \omega_{n} \cdot \mathbf{q}<0\right)$ but rather to its time-reversed counterpart $\left(\mathbf{H}_{\mathbf{k}, n}\right)^{*}$ we define the circular dichroism index

$$
C=\operatorname{sgn}\left(\mathbf{q} \cdot \nabla_{k} \omega\right)\left(C_{\mathbf{k}, n}^{+}-C_{\mathbf{k}, n}^{-}\right) /\left(C_{\mathbf{k}, n}^{-}+C_{\mathbf{k}, n}^{+}\right),
$$

where sgn denotes the sign function [31].

While the phenomenon of circular polarization is evident for a plane wave, it is more subtle for Bloch modes, manifested by the continuous nature of $C$. We call a band RCP if $C>0.5$ and LCP if $C<-0.5$. In addition to the dichroism index that measures the relative coupling of the two circular polarization directions, the coupling index $\beta=\left(C_{\mathbf{k}, n}^{+}+C_{\mathbf{k}, n}^{-}\right) / 2$ measures twice the average degree of coupling between an incident plane wave of arbitrary polarization and a Bloch mode. Qualitatively, $\beta$ provides the probability that a photon represented by a plane wave can propagate into a semi-infinite $\mathrm{PC}$ by coupling to the Bloch mode $\mathbf{H}_{\mathbf{k}, n}$, whereas $C^{+}$and $C^{-}$give this probability for a RCP and LCP photon, respectively. A mode with $\beta<0.1$ is called low coupling.

This analysis reveals two distinct origins for band gaps. First, RCP [LCP] light cannot propagate into an (infinite) PC if no RCP [LCP] band exists, leading to a partial circular dichroic band gap. Second, even if an RCP [LCP] band exists, coupling may be so weak that effectively no light can enter the crystal, leading to a (LCP/ RCP) low-coupling stop band.

Single srs in butterfly wing scales.-Figure 2 shows results for a RH 1srs net, inflated to form a chitin-filled constant mean curvature (CMC) domain with $\Phi=20.8 \%$ [32], for $\mathbf{k}$ in [100]. The band structure reveals a conventional partial band gap for unpolarized light, for $\left[\omega_{1}, \omega_{2}\right] ; c$ is the speed of light in vacuum and $a$ the cubic lattice parameter. The range $\left[\omega_{2}, \omega_{3}\right]$ is a LCP low-coupling stop band. The range $\left[\omega_{3}, \omega_{4}\right]$ is a RCP dichroic band gap and a LCP low-coupling stop band.

Figure 3 shows the widths of the band gaps for the 1srs along [100] as a function of $\Phi$ : A narrow unpolarized conventional band gap exists, which is widened towards higher frequencies by an RCP dichroic band gap and an unpolarized low-coupling gap. Hence, the partial band gaps of this structure are significantly enlarged for light whose circular polarization orientation matches the handedness of the helical twist of the chitin domains.

Quadruple srs materials._Artificial but feasible materials containing multiple CMC gyroids, wrapped on srs nets (all, say, LH), display even more sensitivity to circularly polarized light. The 4srs material with cubic

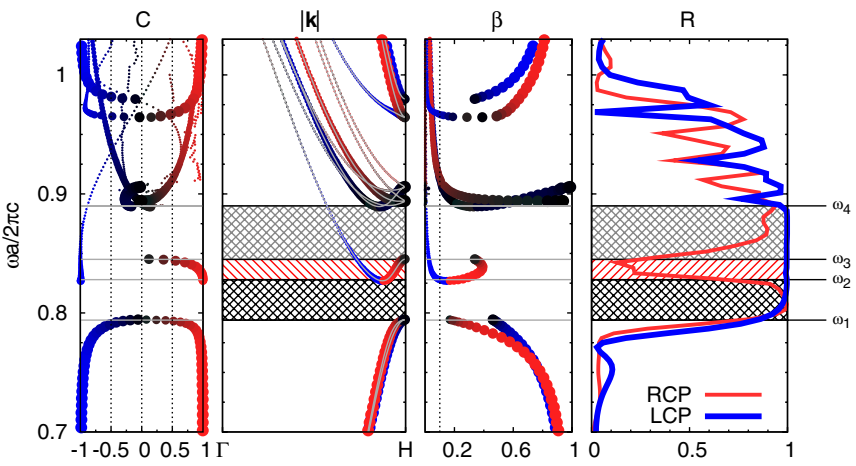

FIG. 2 (color online). Polarization analysis of the band structure of the 1srs structure with $\Phi=20.8 \%$ of the chitin domain $(\epsilon=2.4)$ in vacuum $(\epsilon=1)$. (a) Circular dichroism index $C$; (b) Dispersion relation $\omega(k)$ in [100], from $\Gamma$ to $H$ point; (c) Coupling index $\beta$. The point color represents the value of $C$ and the point radius the value of $\beta$. Vertical dashed lines give the thresholds above which bands are considered LCP (blue [dark gray]), RCP (red [medium gray]) or low coupling. (d) Reflectance rate $R$. The color bar represents the color of light if $a=310 \mathrm{~nm}$ (as in C. rubi). 


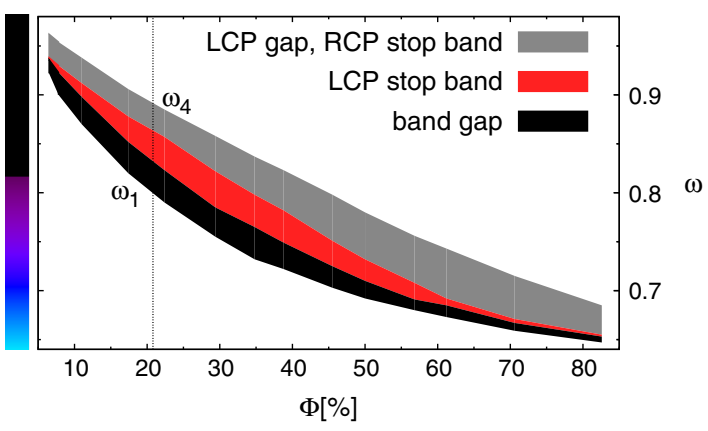

FIG. 3 (color online). Partial gap map $\omega(\Phi)$ for the fourfold screw axis of the 1srs chitin structure. The color bar shows the color of light for a lattice constant of $a=310 \mathrm{~nm}$. The vertical dashed line marks the volume fraction of Fig. 2.

symmetry $\mathrm{P}_{2} 32$ has four interwoven LH srs nets [33]; each net is swollen to form a CMC domain with $\epsilon=12$ (typical semiconductor) and has $\Phi=6 \%$; the background (air) has $\epsilon=1$ (Fig. 4). The photonic band structure (Fig. 5) reveals a very large RCP dichroic band gap in [100], parallel to the four-screw axes. Its relative width is $\Delta:=\frac{2\left(\omega_{2}-\omega_{1}\right)}{\omega_{2}+\omega_{1}}=30.2 \%$ (see Fig. 5), larger than that found for a 1D hexagonal array of vertical helices $(\Delta=26 \%$, [27]). In stark contrast, no band gap (or at most a very narrow one not relevant for PCs with a small number of unit cells) is expected for LCP light. For lattice parameter $a=155 \mathrm{~nm}$ in $P 4_{2} 32$ (i.e., the length scale of the srs nets in C. rubi) it reflects RCP light in a large range of the visible spectrum (Fig. 5). The 4srs is hence a broadband dichroism filter of cubic symmetry, for white light incident along all [100] directions.

Discussion.-This work is motivated by the remarkable synthetic capabilities of butterfly pupae, that manufacture the 1srs chitin scaffold using only protein, lipids, water and chitin precursors [34]. Experimental realizations of multiple srs nets have not been reported to date; however,

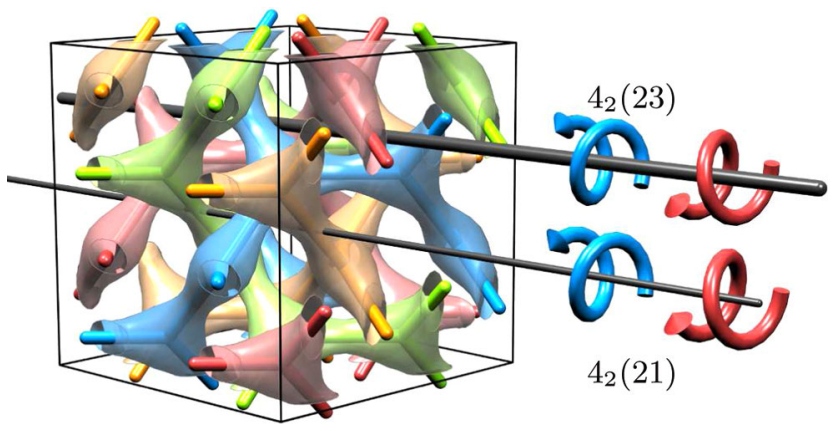

FIG. 4 (color online). 4srs CMC structure with two distinct four-screw axes along [100]. $2^{3}$ unit cells of $P 4_{2} 32$ [17] are shown (individual nets are LH). As a RH turn, the $4_{2}(23)$ screw leaves the two nets that coil around it (blue [dark gray] \& red [medium gray]) unchanged but exchanges the other pair (green [medium light gray] \& orange [light gray]); a LH turn does the opposite. a hexagonal mesoporous silicate with a related morphology has been synthesized [35]; this polycontinuous material contains three interwoven "etc" nets, also called $(\mathbf{8 , 3})$-c [15], an alternative degree-3 network topology to srs. Further, direct laser writing allows the fabrication of prescribed geometries with submicron resolution [36,37], benefiting from the structural stability of srs nets. The study of these srs-based PCs is therefore more than an intellectual exercise, affording eminently realizable designs.

The origin of circular dichroism in these chiral patterns can be comprehended by careful scrutiny. For the 1srs structure the origin of circular dichroism is the difference of the embedding of the two parallel four-screw axes of different handedness. The helical spirals of the highdielectric srs domains coil around the LH screw axis. The parallel RH screw axis of $I 4_{1} 32$ is the analogue for the void domain with opposite rotation sense; for the chitin domain this RH screw is an isometry of less local nature (much wider spiral radius).

The band gap in the 4srs pattern arises despite the fact that there are two distinct four-screw axes in [100], $4_{2}(21)$ and $4_{2}(23)$, each simultaneously LH and RH, see Fig. 4. Application of the $4_{2}(21)$ screw axis cyclically exchanges the four nets (as in an achiral twofold woodpile structure), equally for both rotation senses. The RCP field of the lowest band 1 concentrates its electric energy around the axis in the same way as the LCP field of band 3, hence not inducing circular dichroism. In contrast, for the $4_{2}(23)$ axis, the LH turn is topologically different from the $\mathrm{RH}$ turn, inducing circular dichroism. Two of the nets form a double helix around this axis, with lattice parameter $a$ equal to half the pitch of each helix: The RH turn is a locally topologically continuous screw rotation of each of these two nets whereas the LH turn exchanges them. The wavelength of the dominant Fourier component with Bragg order $(0,0,0)$ matches the spiral pitch at the Brillouin zone edge, inducing a large difference in frequencies of the two

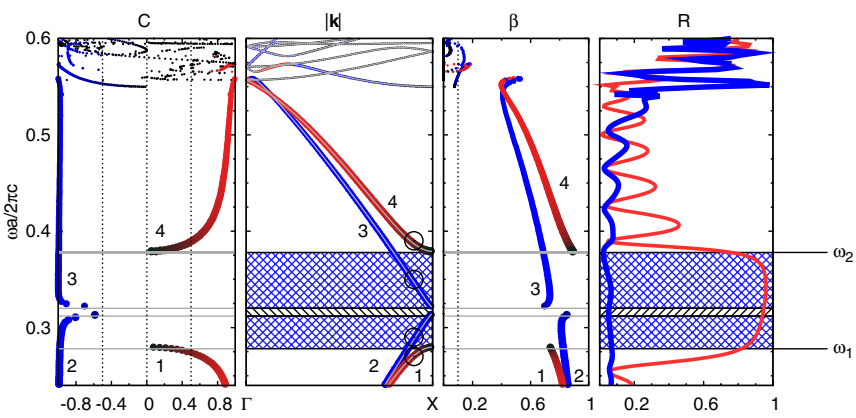

FIG. 5 (color online). Band structure of the 4 srs geometry with $\Phi=24 \%$ for $\mathbf{k}$ along [100]. The relative width of the dichroic band gap is $\Delta=30.2 \%$. Open black circles mark the modes for which electric field energies are calculated (page 4). The oscillations in the reflectance $R$ above $\omega_{2}$ are likely due to the finite number of 6 unit cells in the scattering matrix calculation. The color bar represents the color of light if $2 a=310 \mathrm{~nm}$. 
lowest bands for RCP/LCP light. The time-frozen electric field of band 1 is RCP and hence matches the rotation sense of the double helix. The band arranges its electric field parallel to the interface concentrating the field energy in the dielectric (cf. [38], p. 68). However, band 2 is orthogonal to band 1 so that the dominant Fourier component is LCP and cannot globally arrange its field parallel to the surface. More precisely, far from the screw axes the field of band 3 is similar to band 1 , with an interchanged sense of rotation. Both concentrate field energy in the dielectric around the $4_{2}(21)$ axis, but the RCP mode of band 1 additionally concentrates energy in the spirals around the $4_{2}(23)$ axis. The fraction of field energy in the dielectric $\int_{\varepsilon=12} d^{3} r \varepsilon(\mathbf{r})|\mathbf{E}(\mathbf{r})|^{2} / \int d^{3} r \varepsilon(\mathbf{r})|\mathbf{E}(\mathbf{r})|^{2}$ for $k a / 2 \pi=0.45$ in [100] (calculated near the $X$ point, marked in Fig. 5 by circles) is $59 \%$ for band 1, $47 \%$ for band 2 and 3, and $23 \%$ for band 4 , confirming this geometric picture.

The circular polarization analysis of the band structure is in good agreement with scattering matrix results, with discrepancies in the vicinity of band edges. Our method can be further improved by realizing that backscattered plane waves may also couple to all Bloch modes with $\mathbf{k} H \mathbf{q}$ of the same frequency $\omega$ as the incident wave. At greatly increased computational cost, reflectivity could be obtained by the amplitudes of the resulting scattering problem with suitable electromagnetic boundary conditions at the air-PC interface.

In conclusion, we have shown that biomimetic photonic crystals inspired by chiral srs nets found in butterflies show strong circular dichroism, relevant for insect vision, biophotonics and metamaterial design. The study of the relationship between band structure and propagation properties can shed light on the interplay of physics and geometry that is the origin of these phenomena.

We thank A.-L. Robisch and R. Pinzer for butterfly specimens and G. von Freymann for advice. We acknowledge support by the German Science Foundation through the "Engineering Advanced Materials" Cluster Erlangen, and by the Australian Research Council through CUDOS.

*Gerd.Schroeder-Turk@physik.uni-erlangen.de

[1] A. Schoen, Infinite Periodic Minimal Surfaces without Self-Intersections, Tech. Rep. (NASA, Washington, DC, 1970).

[2] K. Michielsen and D. Stavenga, J. R. Soc. Interface 5, 85 (2008).

[3] S. T. Hyde, M. O'Keeffe, and D. Proserpio, Angew. Chem., Int. Ed. Engl. 47, 7996 (2008).

[4] V. Saranathan et al., Proc. Natl. Acad. Sci. U.S.A. 107, 11676 (2010).

[5] P. Vukusic and J. Sambles, Nature (London) 424, 852 (2003).

[6] G. Horváth and D. Varjú, Polarized Light in Animal Vision (Springer, Berlin, 2003).
[7] S. Kleinlogel and A.G. White, PLoS ONE 3, e2190 (2008).

[8] V. Sharma, M. Crne, J. Park, and M. Srinivasarao, Science 325, 449 (2009).

[9] A. Sweeney, C. Jiggins, and S. Johnsen, Nature (London) 423, 31 (2003).

[10] O. Toader and S. John, Science 292, 1133 (2001).

[11] M. Thiel et al., Opt. Lett. 35, 166 (2010).

[12] J. Gansel et al., Science 325, 1513 (2009).

[13] O. Delgado-Friedrichs, M. O'Keeffe, and O. Yaghi, Acta Crystallogr. Sect. A 59, 22 (2003).

[14] H. Heesch and F. Laves, Z. Kristallogr. 85, 443 (1933); A. Wells, Acta Crystallogr. 7, 535 (1954).

[15] A. Wells, Three-Dimensional Nets and Polyhedra (Wiley \& Sons, New York, 1977).

[16] The network's single type of 3-coordinated vertices are at Wyckhoff sites $8 a(.32)$ in $I 4_{1} 32$ (no. 214 in [17]).

[17] International Tables For Crystallography, edited by T. Hahn (Kluwer Academic Publishers, Dordrecht, 1992).

[18] G. Pringle, Acta Crystallogr. Sect. B 28, 2326 (1972).

[19] K. Grosse-Braukmann, J. Colloid Interface Sci. 187, 418 (1997).

[20] J. Sun et al., Nature (London) 458, 1154 (2009).

[21] T. Epps et al., Macromolecules 37, 8325 (2004).

[22] C. Kepert, T. Prior, and M. Rosseinsky, J. Am. Chem. Soc. 122, 5158 (2000).

[23] S. T. Hyde and C. Oguey, Eur. Phys. J. B 16, 613 (2000).

[24] S. T. Hyde and G. E. Schröder, Curr. Opin. Colloid Interface Sci. 8, 5 (2003).

[25] S. T. Hyde et al., Solid State Sci. 5, 35 (2003).

[26] S. T. Hyde, L. De Campo, and C. Oguey, Soft Matter 5, 2782 (2009).

[27] J. C. W. Lee and C. T. Chan, Opt. Express 13, 8083 (2005).

[28] D. M. Whittaker and I. S. Culshaw, Phys. Rev. B 60, 2610 (1999).

[29] A.F. Oskooi et al., Comput. Phys. Commun. 181, 687 (2010).

[30] S. Johnson and J. Joannopoulos, Opt. Express 8, 173 (2001).

[31] The sign of the group velocity alleviates the necessity to identify the dominant Fourier component of Ref. [27]; the relative difference is more robust than the quotient.

[32] Band structures are calculated by MPB with $512^{3}$ voxels for real-space discretization of $\epsilon(\mathbf{r})$ (derived from CMC triangulations by Surface Evolver [19]) and $64^{3}$ grid for Fourier space. $R$ is calculated on six unit cells in the propagation direction with $128^{3}$ voxels per cubic unit cell.

[33] The vertices are at Wyckhoff sites $4 b$ (.32) and edge midpoints at $6 f(2.22)$ in $P 4_{2} 32$ (no. 24 in [17]). The lattice parameter $a$ is half that of the $I 4_{1} 32$ 1srs.

[34] H. Ghiradella and W. Radigan, J. Morphol. 150, 279 (1976).

[35] Y. Han et al., Nature Chem. 1, 123 (2009).

[36] K. K. Seet et al., Appl. Phys. Lett. 88, 221101 (2006).

[37] M. Thiel et al., Adv. Mater. 21, 4680 (2009).

[38] J. Joannopoulos et al., Photonic Crystals: Molding the Flow of Light (Princeton University Press, Princeton, NJ, 2008).

[39] See supplemental material at http://link.aps.org/ supplemental/10.1103/PhysRevLett.106.103902 for animation. 